\title{
L'interface de l'homme et de la machine et la fiabilité humaine
}

Par M. WANNER, Ingénieur Général de l'Air.$$
\text { I y a une quinzaine d'années, j'ai }
$$
été chargé de rédiger le règlement de certification de Concorde. Lórsque, avec l'équipe qui travaillait avec moi, dont certains membres sont ici aujourd'hui, nous nous sommes attaqués au problème, nous nous sommes rendus compte très rapidement que les règlements existants et applicables aux avions du type Boeing 707 ne s'appliquaient absolument pas à Concorde car les conditions d'utilisation de Concorde étaient vraiment trop différentes d'un Boeing 707 ou d'une Caravelle. Nous avons donc été amenés à regarder dans quelles conditions se produisait un accident, de façon à essayer de bâtir, de façon plus rigoureuse, ce fameux règlèment. Nous en sommes arrivés à la conclusion qu'un accident se produit lorsque le point de fonctionnement de l'appareil dépasse une certaine limite.

Qu'appelle-t-on point de fonctionnement ?

Chacun des composants du grand système qu'est un avion, peut être caractérisé par un certain nombre de paramètres et chacun de ces paramètres doit rester à l'intérieur de certaines limites. On peut donc représenter le fonctionnement de l'ensembe du système par un point dans un espace à $n$ dimensions et lorsque le point de fonctionnement dépasse une des limites, nous pouvons dire qu'il y a catastrophe.

Bien entendu, cette notion de limites est un peu floue ; il faut en réalité atta- cher une probabilité d'accident à chaque point de fonctionnement, cette probabilité étant d'autant plus voisine de 1 que l'on est proche d'une limite.

Mais on peut, pour simplifier les choses, parler d'un domaine où tout se passe bien, et admettre qu'il y a catastrophe au franchissement d'une limite.

La question que l'on peut alors se poser est pourquoi y a-t-il franchissement de limites?

Le point de fonctionnement se déplace à l'intérieur du domaine, à la suite d'une succession d'incidents, et chacun de ces incidents peut être classé dans l'une des trois catégories suivantes et trois catégories seulement.

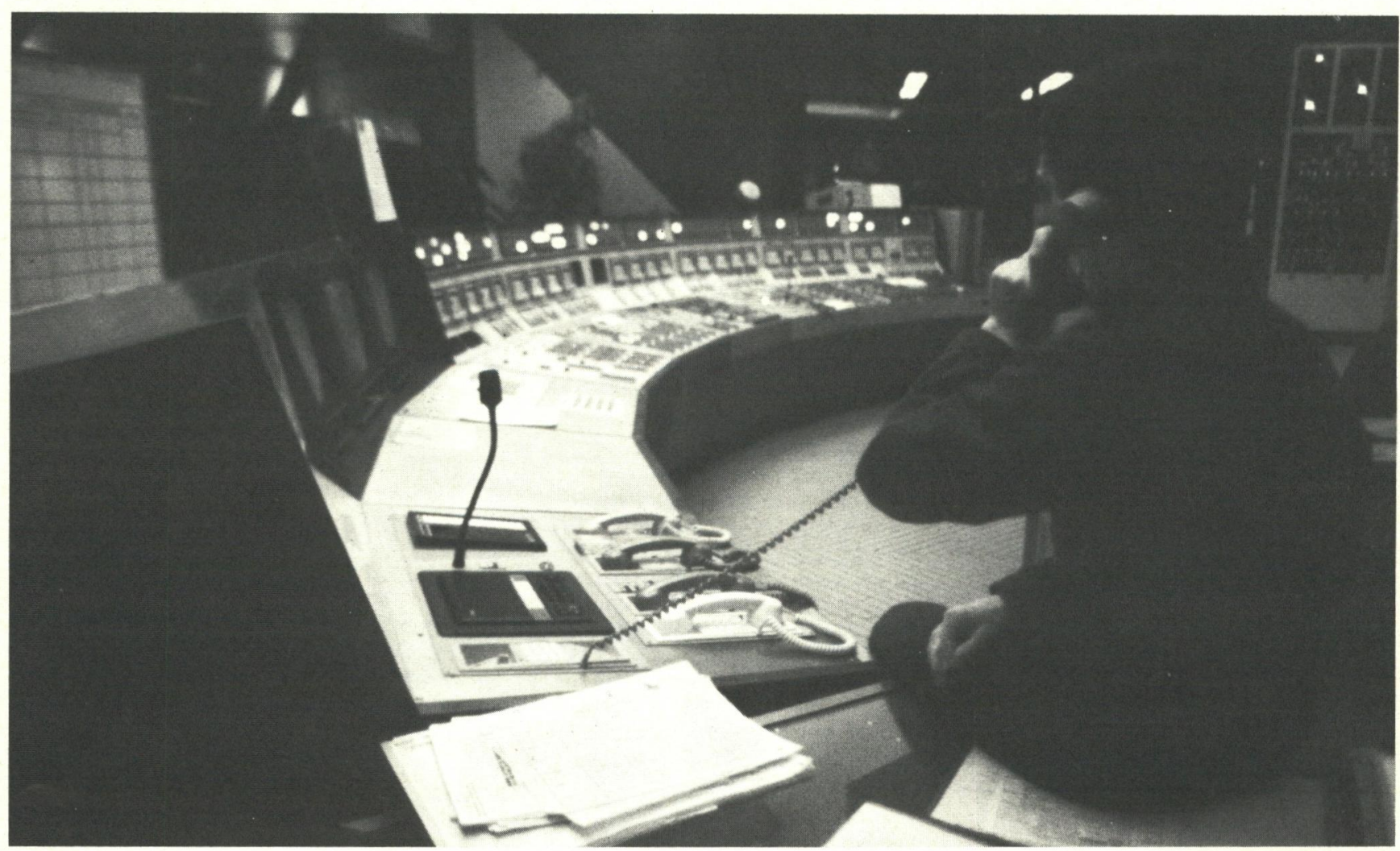

Dans la salle de contrôle de la centrale nucléaire du Tricastin. 
- Le premier type d'incident est l'incident que nous avons qualifié d'incident de pilotabilité dans lequel intervient l'opérateur humain. Un incident de pilotabilité se présente de la façon suivante : on demande à l'opérateur d'effectuer trop d'opérations d'actions sur les commandes, trop d'opérations d'interprétation des données. L'opérateur débordé, oublie alors l'une des opérations ou l'exécute mal. En conséquence, le point de fonctionnement se déplace; ce qui ne veut pas dire que l'on a automatiquement une catastrophe, mais l'incident déplace le point de fonctionnement, peutêtre en direction d'une limite.

- Le deuxième type d'incident est l'incident de sensibilité aux perturbations. A la suite de perturbations externes, pour un avion une rafale, pour une automobile une ondulation de la route ou d'une perturbation interne, apparition d'une panne, le point de fonctionnement se déplace, peut-être en direction d'une limite, ou bien c'est une limite qui se déplace en direction du point de fonctionnement. Dans ce dernier cas en effet lorsqu'une panne apparaît, l'état du système est changé, et la position de la limite autorisée peut se déplacer.

Nous voilà donc en présence des deux premiers types d'incidents, incidents de pilotabilité faisant intervenir l'homme, incident de sensibilité aux perturbations, ne faisant pas intervenir l'homme, et faisant intervenir, par contre, le milieu extérieur, ou bien faisant intervenir les situations de panne possible. II faut noter que nous parlons bien d'apparition de panne et non pas de panne établie. Lorsque l'on se trouve avec un système avec une panne établie, on est en présence d'un nouveau système, qu'il faut étudier en tant que tel.

- Enfin, le troisième type d'incident, plus difficile à décrire, est l'incident que nous appellerons incident de manœurrabilité. A la suite d'incidents des deux premiers types, le point de fonctionnement s'est déplacé, et moyennant quoi, l'opérateur est amené à effectuer une manœuvre pour ramener le point de fonctionrement à la position nominale. Ou encore, pour remplir la mission du système, l'opérateur est amené à effectuer une manœuvre. Par exemple, lorsque l'automobiliste se trouve aborder un virage, pour remplir la mission de l'automobile qui consiste à maintenir la voiture sur la route, l'opérateur est amené à effectuer une manœuvre de changement de direction. Au cours de chaque manœuvre, il y a déplacement du point de fonctionnement. On va donc décrire une petite courbe à l'intérieur du domaine de fonctionnement, et à l'occasion de ce déplacement, le point de fonctionnement peut se rapprocher d'une limite.
Lorsqu'on analyse les systèmes, on constate qu'il n'y a que ces trois types d'incidents qui peuvent conduire au déplacement du point de fonctionnement et c'est à la suite d'une succession d'incidents de ces trois types, que l'on peut rencontrer effectivement l'accident.

es règles de sécurité ont donc pour but de diminuer les probabilités de déplacement du poin de fonctionnement en direction des limites pour chacun de ces types d'incidents. II est bien évident que les règles de sécurité ne vont pas être les mêmes pour protéger le point de fonctionnement d'un déplacement intempestif, s'il s'agi de règles relatives à un problème de pilotabilité, à un problème de manœuvrabilité ou à un problème de sensibilité aux perturbations.

Le problème de sensibilité aux perturbations, le problème de manœuvrabilité, peuvent-être traités de façon puremen mécanique. II suffit de connaître le fonctionnement mécanique du système, de connaître l'environnement et d'étudier par le calcul le fonctionnement du système pour se convaincre d'un certain nombre de valeurs numériques de probabilités d'accidents.

Par contre, en ce qui concerne le problème de pilotabilité, intervient le facteur humain. Jusqu'à présent, dans l'étude des systèmes, on s'en est tenu aux deux .critères de sensibilité aux perturbations et de manœuvrabilité et l'on a eu quelque peu tendance à oublier les incidents de pilotabilité. Maintenant, ayant traité les deux types d'incidents manœuvrabilité et sensibilité aux perturbations, il nous faut traiter les incidents de pilotabilité de façon à avoir des règles de sécurité homogènes. Pou cela, on fait appel à l'ergonomie. La encore, on a tendance à ne regarder qu'une partie des aspects ergonomiques.

La première des choses que l'on regardé jusqu'ici est ce que nous appellerons l'ergonomie mécanique. On met l'opérateur humain en position vis-à-vis de la machine et l'on regarde s'il est capable d'atteindre les diverses commandes, d'observer les divers cadrans s'il est suffisamment bien éclairé, s'i peut entendre correctement les informations, etc. En général, ces études son très correctement menées. II existe maintenant d'ailleurs de nombreuses banques de données qui permettent d'aider l'ingénieur à concevoir clairement les postes de commande.

La deuxième chose à être égalemen étudiée est l'aspect médical de l'opérateur, appelons ça l'aspect physiologi- que. On regarde si l'opérateur, en condition de travail sur sa machine, se trouve dans des conditions physiologiques suffisamment correctes pour lui permettre de survivre. On regarde donc les problèmes d'ambiance, de température, de pression, de vibrations, d'éclairage, de bruits, etc., de façon à vérifier que l'opérateur dans ces conditions ne risque pas de souffrir physiologiquement de l'ambiance. Là encore ce genre d'étude commence à être faite de façon correcte.

Mais il reste un troisième domaine à traiter et celui-ci pour l'instant, laisse encore à désirer : c'est le problème de ce que j'appellerai l'ergonomie mentalle. Une fois l'opérateur installé confortablement dans un fauteuil, sans vibrations, sans bruits, avec une température correcte, avec de la moquette, et de nombreux instruments, avec une multitude de commandes qu'il peut atteindre très facilement, on est convaincu que le problème est résolu. L'opérateur n'a plus qu'à faire ce qu'on lui dit.

Or, en général on n'a pas fait attention à l'aspect psychologique. Nous savons tous qu'il est difficile de faire marcher un homme sur les mains. C'est possible mais la probabilité pour qu'il tombe est nettement supérieure au cas où on le fait marcher sur les pieds. On sait par ailleurs qu'il n'aime pas beaucoup ça mais qu'il peut y arriver par entraînement. On sait toutefois que ses performances ne seront pas très bonnes. Dans ces conditions on tient compte des risques.

Par contre, on met un opérateur devant une multitude de cadrans, qui n'indiquent rien, parce que figés par les automatismes et on demande à cet opérateur de passer huit heures dans une ambiance feutrée, agréable, à la bonne température, en attendant une panne qui n'arrive peut-être jamais ou qui n'arrive toujours qu'aux autres. II faut alors se poser le problème, si psychologiquement, l'opérateur humain est prêt à travailler dans ces conditions. En réalité, l'homme n'a pas tellement évolué depuis l'époque du chasseur-cueilleur; psychologiquement, il fonctionne toujours comme l'homme de Cromagnon, et il faut faire attention à ne pas lui imposer des tâches complètement contraires à ses habitudes. Lorsque l'homme fabriquait ses outils lui-même, il savait se les adapter. Maintenant, c'est quelqu'un d'autre qui construit l'outil et qui impose à l'opérateur de travailler avec ce nouvel outil. On a su adapter l'outil à la morphologie de l'homme, adapter l'outil à la physiologie de l'homme. II faut maintenant penser à adapter l'outil à la psychologie de l'homme. Je suppose que c'est là un des points les plus importants que nous avons à traiter. 